\title{
Label-Free Biosensing Based on Multilayer Fluorescent Nanocomposites and a Cationic Polymeric Transducer
}

\author{
Danny Brouard, ${ }^{\dagger}$ Mathieu L. Viger, ${ }^{\dagger}$ A. Guillermo Bracamonte, and Denis Boudreau*
}

Département de chimie and Centre d'optique, photonique et laser (COPL), Université Laval, Québec (QC), Canada G1V 0A6. ${ }^{\dagger}$ These authors contributed equally to this work.

$S$ ensitive fluorescence-based DNA detection techniques are widely used in forensic analyses, ${ }^{1}$ diagnosis of infectious diseases, ${ }^{2,3}$ and identification of genetic mutations, ${ }^{4-6}$ biological pathogens, ${ }^{7}$ and environmental contaminants. ${ }^{8}$ Because of the limited levels of targets present in a particular sample, one often relies on the enzymatic amplification of target DNA to achieve the required sensitivity. ${ }^{9-11}$ However, such a procedure is time-consuming, laborious, prone to contamination, and induces high levels of complexity and cost. $^{12-14}$ To avoid the added complexity of enzyme-based approaches, complementary strategies are being developed to instead amplify the optical signal generated by each target molecule detected. ${ }^{15,16}$ Some among those new strategies employ single-stranded DNA (ssDNA)-decorated fluorescent nanoprobes such as semiconductor quantum dots and dye-doped silica nanoparticles (NP) to provide enhanced detection response as compared to that of individual molecular fluorophores as well as improved robustness toward photobleaching. ${ }^{17-20}$

A new class of fluorescent NPs recently emerged as promising optical tools for a variety of applications including ultrasensitive DNA detection. Multilayer metal coresilica shell fluorescent NPs offer several advantages over commonly used dyedoped nanoparticles. ${ }^{21-27}$ For example, the interaction of dye molecules with the metal core greatly improves the excitation efficiency, enhances the emissive rates, and reduces the lifetime of excited states, resulting in enhanced photostability and detectability. Furthermore, self-quenching is suppressed by plasmonic interaction via the strong decrease in nonradiative
ABSTRACT This study describes the preparation and characterization of a DNA sensing architecture combining the molecular recognition capabilities of a cationic conjugated polymer transducer with highly fluorescent core-shell nanoparticles (NPs). The very structure of the probelabeled NPs and the polymer-induced formation of NP aggregates maximize the proximity between the polymer donor and acceptor NPs that is required for optimal resonant energy transfer. Each hybridization event is signaled by a potentially large number of excited reporters following the efficient plasmon-enhanced energy transfer between target-activated polymer transducer and fluorophores located in the self-assembled core-shell aggregates, resulting in direct molecular detection of target nucleic acids at femtomolar concentrations.

KEYWORDS: fluorescence enhancement - resonant energy transfer - plasmonic . silver core-silica shell nanoparticles · DNA sensing

molecular decay rates, which allows the incorporation of larger amounts of dye molecules into the silica shell, ${ }^{21,28}$ while the latter protects the fluorophores from collisional quenching and provides easy conjugation to biomolecular probes such as oligonucleotides and antibodies. Another benefit of plasmonic enhancement in coreshell nanostructures is the improvement of FRET (Förster resonant energy transfer) efficiency, range, and transfer rate. ${ }^{22,29-32}$ As a consequence, the location of donor and acceptor molecules in proximity to the metal core increases the strength of donoracceptor interactions and leads to the excitation by a single donor of several acceptor molecules over distances exceeding the natural range of FRET. Therefore, the use of plasmonic enhancement in the development of a FRET-mediated DNA detection scheme can lead to massive improvement in signal amplification and sensing capacity.

In this paper, we report the development of a novel plasmonic- and FRET-based DNA sensing scheme based on core-shell @2011 American Chemical Society 

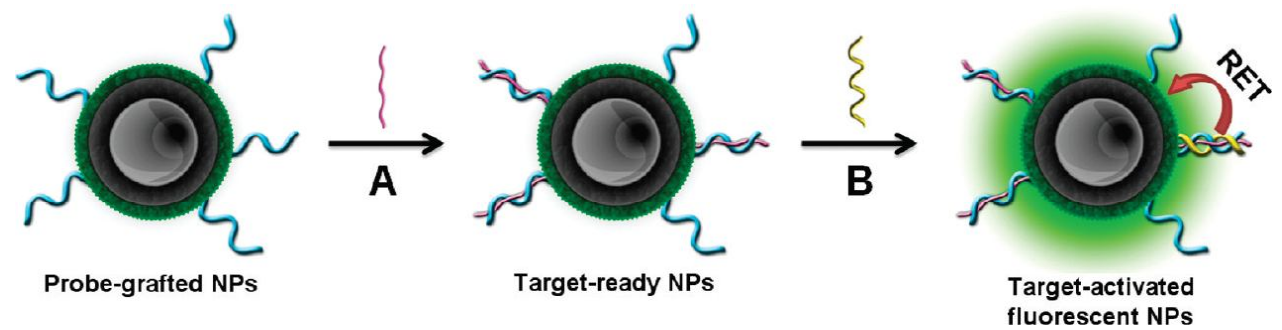

Figure 1. Principle of DNA detection on fluorescent multilayer core-shell NPs: (a) target-ready NPs are prepared by complexing ssDNA probe-grafted NPs with polymer transducer; (b) hybridization of target DNA with ssDNA probes activates the polymer transducer as energy donor toward dye-doped silica shell and excitation at $410 \mathrm{~nm}$ generates fluorescence emission by acceptor molecules at $550 \mathrm{~nm}$.
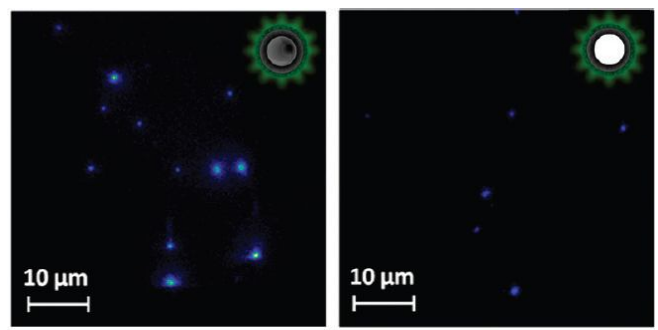

Intensity scale (counts)

2500

40000

Figure 2. Imaging flow cytometry (IFC) images recorded from Ag@SiO $@_{2} \mathrm{SiO}_{2}+$ EiTC nanocomposites (left) and hollow nanoshell control samples (right). The excitation wavelength was $488 \mathrm{~nm}$, and the fluorescence emission was collected through a $528 \pm 19 \mathrm{~nm}$ band-pass filter. See Supporting Information for experimental details.

multilayer dye-doped acceptor nanoparticles grafted with ssDNA probes and complexed with a cationic conjugated polymer (CCP). This approach exploits the chromism of the CCP, that is, intensity and spectral changes in absorption and luminescence caused by conformational changes of its conjugated backbone upon electrostatic binding to DNA strands. ${ }^{33-35}$ The capture of ssDNA targets onto the surface of the NPs allows the transduction by the CCP of the hybridization event to a FRET-mediated fluorescence signal by allowing the light from a suitable source to be coupled from the polymer to the acceptor molecules immobilized in the outer silica layer of the NPs. ${ }^{16,36-39}$ The capacity of the polymer as energy donor is increased by strong near-field interactions in this plasmonic-enhanced FRET system and results in faster transfer rate and enhanced FRET efficiency and range. In addition, binding of positively charged polymer chains onto negatively charged oligonucleotide-grafted NPs partially neutralizes the particles' surface charge and leads to the formation of NP aggregates. ${ }^{40,41}$ These nanoparticle networks allow collective interactions between NPs which, in turn, enhance plasmonic coupling and local electric field intensities between the NPs ${ }^{42}$ and amplify the overall optical signal generated by DNA hybridization events. The resulting improvement in detection sensitivity allows the development of fast, reliable, and affordable molecular typing procedures without any prior chemical amplification or tagging of the starting sample.

\section{RESULTS AND DISCUSSION}

The new particle-based DNA sensing scheme, illustrated in Figure 1, combines a probe-grafted fluorescent core-shell NP and a CCP (poly $(1 \mathrm{H}$-imidazolium, 1-methyl-3-[2-[(4-methyl-3-thienyl)oxy]ethyl]--, chloride) ${ }^{33}$ in a FRET pair with the polymer acting as the donor and the dye-doped NP as the acceptor. We chose eosin- $5^{\prime}$ isothiocyanate (EiTC) as the energy acceptor because of its well-documented photophysical characteristics and the adequate overlap of its absorption band with the emission band of the polymer donor (Supporting Information, Figure S1). Monodisperse fluorescent multilayer Ag@SiO ${ }_{2} @ S_{2}+$ EiTC NPs with $50 \pm 10$ nm diameter silver cores and uniform $7 \pm 1 \mathrm{~nm}$ thick silica spacer shells were prepared using a procedure published recently (see ref 22, Methods, and Figure S2 in Supporting Information) and adapted for use as the FRET acceptor in the present work. This particular core diameter was chosen based on the report by the Lakowicz group showing that plasmonic enhancement efficiency for fluorophores near the surface of silver nanoparticles reaches a maximum for particle diameters in the $40-70 \mathrm{~nm}$ range, ${ }^{43}$ whereas the $7 \mathrm{~nm}$ spacer thickness was determined in a previous study by our group for $\sim 50 \mathrm{~nm}$ Ag cores using varying silica spacer shells. ${ }^{22}$ The dye concentration in the outer silica shell was chosen to avoid self-quenching between dye molecules and to maximize fluorescence emission. The emission intensity of these core-shell NPs is enhanced by a factor of 10 , as compared with hollow silica nanoshell control samples (Figure 2). This high luminosity, combined with the excellent chemical and optical stability and biocompatible surface of these composite NPs, makes them ideal candidates as nanolabels for sensitive detection of DNA material. The NPs were grafted with amine-modified 20-mer oligonucleotide probes using $N$-hydroxysuccinimide (NHS) cross-linking chemistry, and the surface coverage density of these oligonucleotide-grafted NPs was determined to be $\sim 1500$ oligonucleotides per 

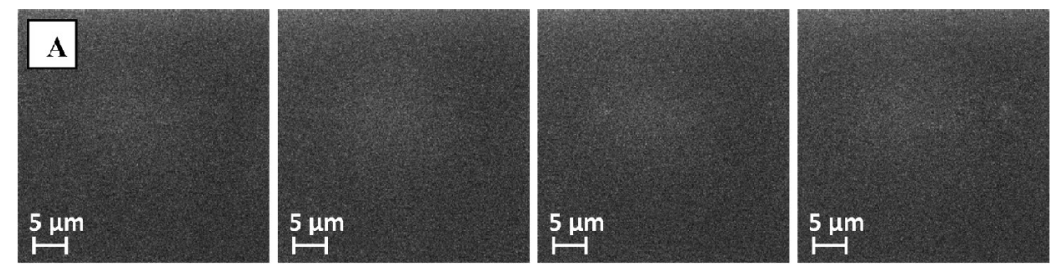

Intensity scale (counts)

2500

8500
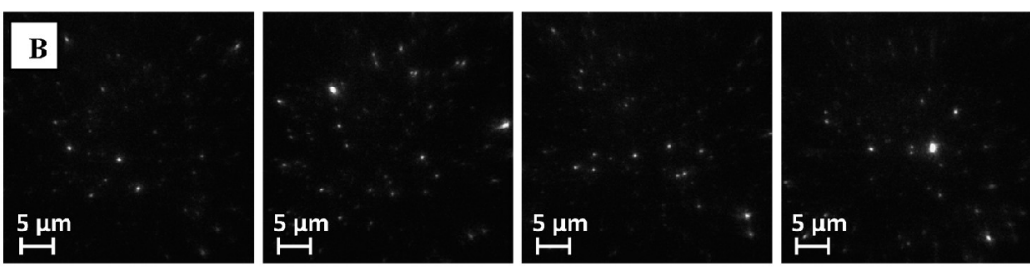

Intensity scale (counts)

2500

10000
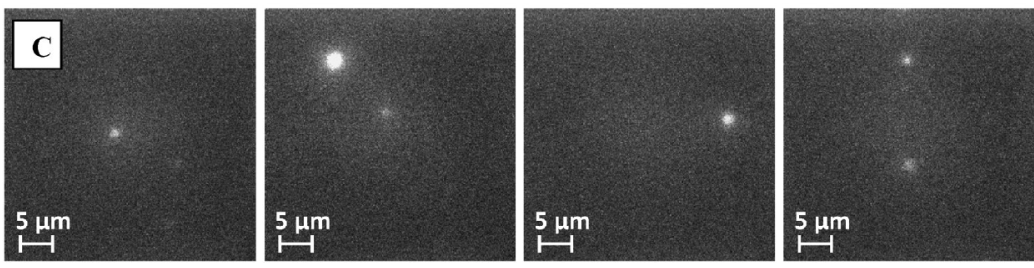

Intensity scale (counts)

2500

8500

Figure 3. IFC fluorescence images, recorded at the nominal emission wavelength of the eosin acceptor, of (A) target-ready NPs without complementary targets, excitation $410 \mathrm{~nm}$; (B) target-ready NPs without complementary targets, excitation 488 $\mathrm{nm}$; (C) images recorded after addition of complementary targets, excitation $410 \mathrm{~nm}$. The fluorescence signal was recorded through a $528 \pm 19 \mathrm{~nm}$ band-pass filter.

nanoparticle (see Methods), which is comparable to coverage densities reported by other groups. ${ }^{44,45}$

When the CCP is added to the NPs, it binds electrostatically to negatively charged ssDNA probes and adopts a planar and highly conjugated form in which fluorescence is effectively quenched. ${ }^{46}$ In the presence of complementary DNA targets, hybridization takes place and the polymer is believed to wrap around the DNA double helix, reverting to a nonplanar, fluorescent conformation, ${ }^{33}$ whereupon excitation of the polymer donor at $410 \mathrm{~nm}$ results in sensitized emission from eosin acceptors located in the outer silica shell via resonant energy transfer from the polymer-dsDNA complexes (Figure 1). The design of this sensing scheme was guided by the prediction that the luminescence of both the polymer and eosin molecules would be strongly enhanced in proximity to the silver core because both have relatively low intrinsic quantum yields ${ }^{47}$ (57\% for eosin ${ }^{48}$ and $3 \%$ for the poly$\mathrm{mer}^{33}$ ) and because the plasmonic band of ${\mathrm{Ag} @ \mathrm{SiO}_{2}}$ nanocomposites (centered at $400 \mathrm{~nm}$ ) overlaps well with the absorption band of the polymer transducer. Recent studies of the influence of plasmonic coupling on the optical properties of different CPs adsorbed onto silver nanoparticles or silver island films have reported changes in the optical properties of the CPs, including enhanced luminescence and shortened excited state lifetimes. ${ }^{49-51}$ This was confirmed in the present work by the measurement of a 30 -fold enhancement in the fluorescence signal with the polymer adsorbed onto $\mathrm{Ag@SiO}_{2} \mathrm{NPs}$ (see Figure $\mathrm{S} 3$ in Supporting Information). Finally, it was also hoped that the interaction of the metal core with both fluorophores would result in reduced lifetime and thus improved photostability, as well as increased efficiency and range of FRET transfer between the polymer donor and the eosin acceptors. ${ }^{22}$

Since the polymer exists in its quenched planar conformation in the target-ready NPs, the latter are nonfluorescent when observed by imaging flow cytometry (IFC) and excited near the nominal excitation wavelength of the polymer donor at $410 \mathrm{~nm}$ (Figure 3A); however, they are easily detectable when excited directly at the nominal excitation wavelength of the eosin acceptor at $488 \mathrm{~nm}$ (Figure 3B). Upon the addition of complementary 20-mer oligonucleotides to the target-ready NPs (Figure 3C), excitation at $410 \mathrm{~nm}$ results in the appearance of objects that are on average significantly larger than on images recorded from probe-grafted NPs before addition of the CCP. 


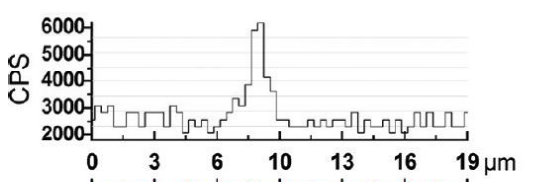

CPS

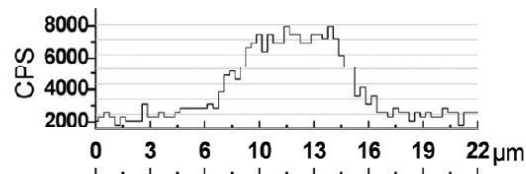

CPS
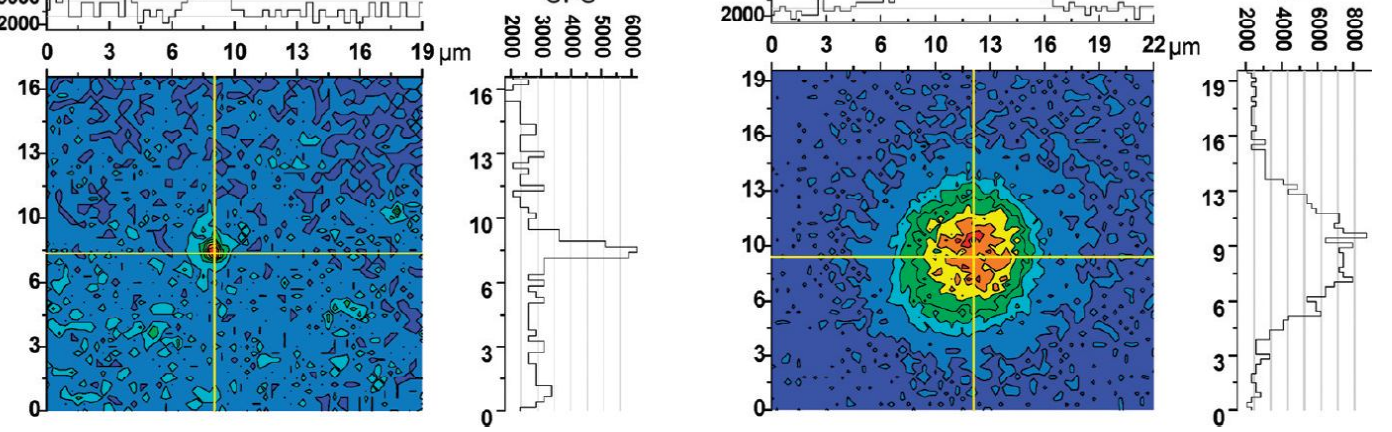

Figure 4. Point spread function images of particles from the two distinct populations of nanoparticle aggregates observed by IFC, following the addition of complementary targets $\left(4.8 \times 10^{-15} \mathrm{M}\right)$ to target-ready NPs.

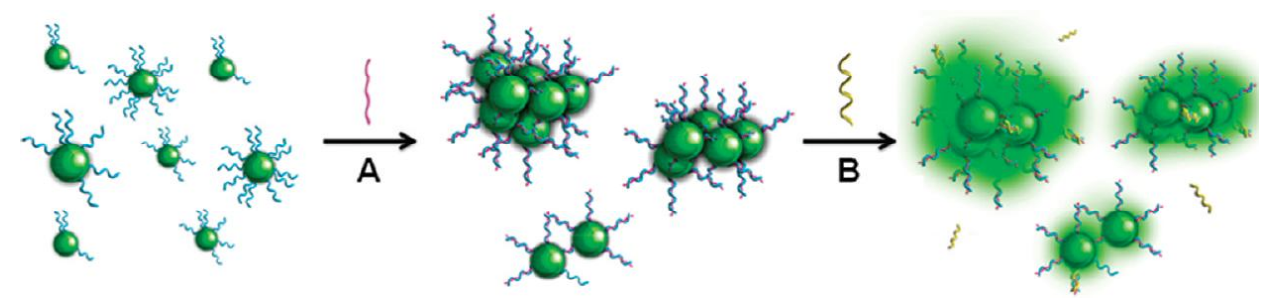

Figure 5. Principle of DNA detection using polymer-induced particle networks: (A) target-ready nanoparticles aggregate following partial charge neutralization by the cationic polymer; (B) hybridization of target oligonucleotides activates polymer transducer as energy donor toward dye-doped silica shell and excitation of polymer at $410 \mathrm{~nm}$ generates emission of fluorescence by acceptor molecules at $550 \mathrm{~nm}$.

Statistical analysis of IFC images based on size, shape, and intensity profile of the recorded objects reveal the presence of two distinct populations (Figure 4). The objects grouped in the first population, which accounts for the majority of all objects detected, are small and generate weak fluorescence signal over a Gaussian intensity profile (Figure 4, left). These objects, too small to be resolved by the IFC apparatus (optical resolution $\sim 1 \mu \mathrm{m}$ ), may correspond to individual NPs or small NP aggregates bearing target oligonucleotides. The objects in the second population are larger $(\sim 2-6 \mu \mathrm{m}$ in diameter) and emit stronger fluorescence signal characterized by a top-hat intensity distribution (Figure 4, right). Despite being less numerous than the smaller objects, statistical image analysis reveals that the integrated emission intensity from these larger objects, which we believe are large aggregates of target-ready NPs, accounts for $\sim 80 \%$ of the total analytical signal (see Figure S9 in Supporting Information).

NPs grafted with anionic oligonucleotides are not expected to aggregate in pure water; ${ }^{52}$ however, the formation of large aggregates of target-ready NPs might arise from the partial neutralization of negative surface charges on probe-grafted NPs following addition of the positively charged polymer transducer, ${ }^{40}$ and hybridization of target molecules onto these larger aggregates might then lead to readily detectable changes in luminosity (Figure 5). Interestingly, a shift toward longer wavelengths of the extinction spectrum of metallic nanoparticles is known to occur with decreasing interparticle distance, ${ }^{53-55}$ and the formation of these aggregates upon the addition of CCP to probegrafted NPs can also be observed by dark-field scattering microscopy as a change in the color of scattered light (Figure 6, left), where the blue or blue-green color of probe-grafted NPs is shifted toward the red upon the addition of the CCP. Furthermore, the increase in size of NP aggregates is obvious in the histograms calculated from the statistical analysis of dark-field scattering images (Figure 6, right).

The sensitive response of NP aggregates to the addition of complementary targets is incumbent on the combination of three processes: (i) recognition and transduction of the hybridization event by the CCP; (ii) plasmonic enhancement of the quantum yield of the CCP and the eosin; (iii) excitation of the eosin-doped silica shell by the polymer transducer. This was verified in separate experiments where either the CCP transducer, silver core, or the eosin acceptor was omitted. In all cases, a dramatic decrease in signal intensity was observed (see Supporting Information, Figure S8).

As illustrated in Figure 7, a linear relationship exists between luminescence intensity and the concentration of target analyte, and it is possible with this molecular sensing system to specifically detect the hybridization of a few thousand molecules of complementary oligonucleotides in a few minutes. In the case of 20-mer target oligonucleotides, the limit of detection was 

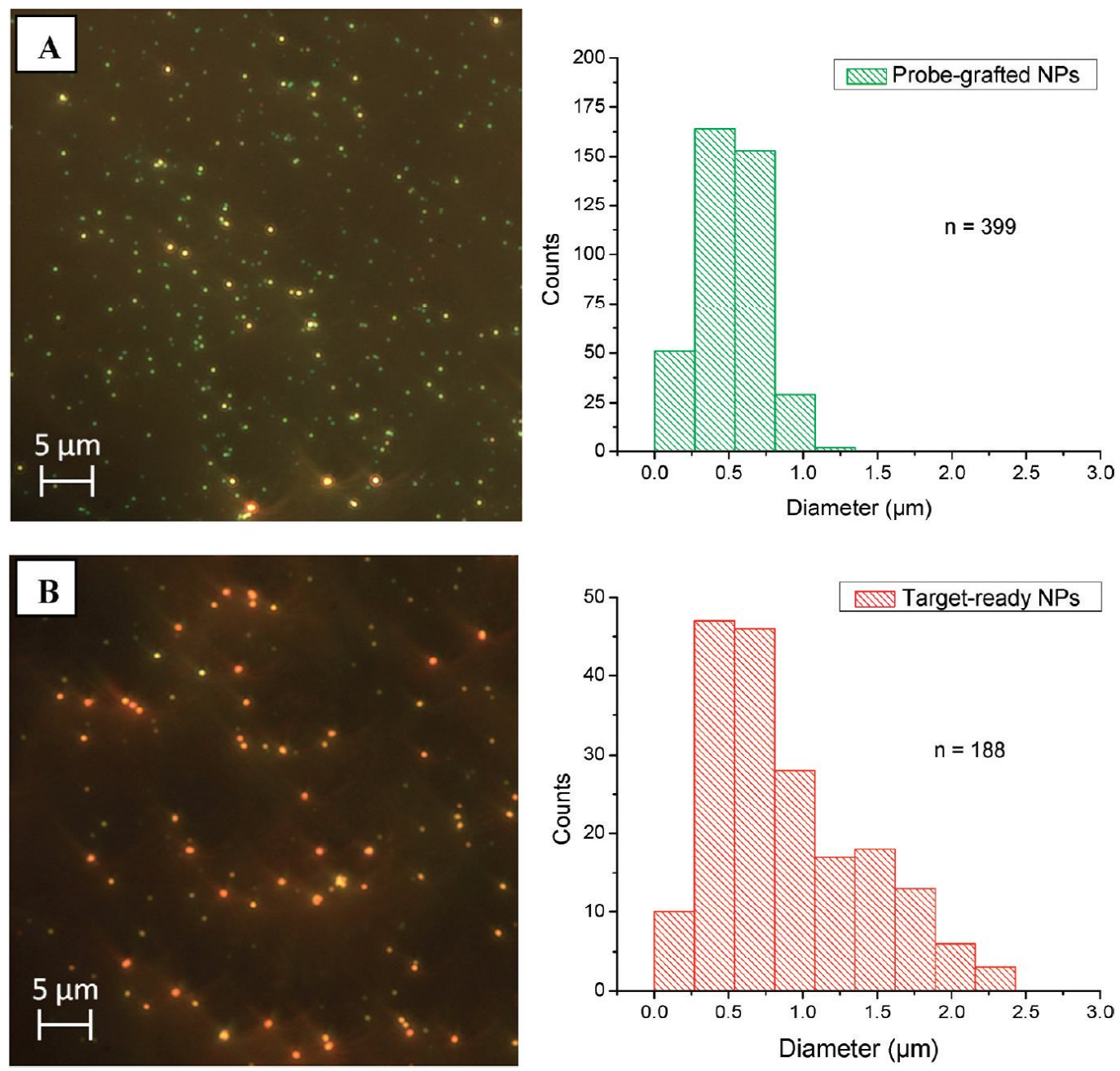

Figure 6. Color images of dark-field scattered light microscopy (left) and corresponding spot size distribution histograms (right) of (A) probe-grafted nanoparticles and (B) target-ready nanoparticle aggregates. See Supporting Information for experimental details.

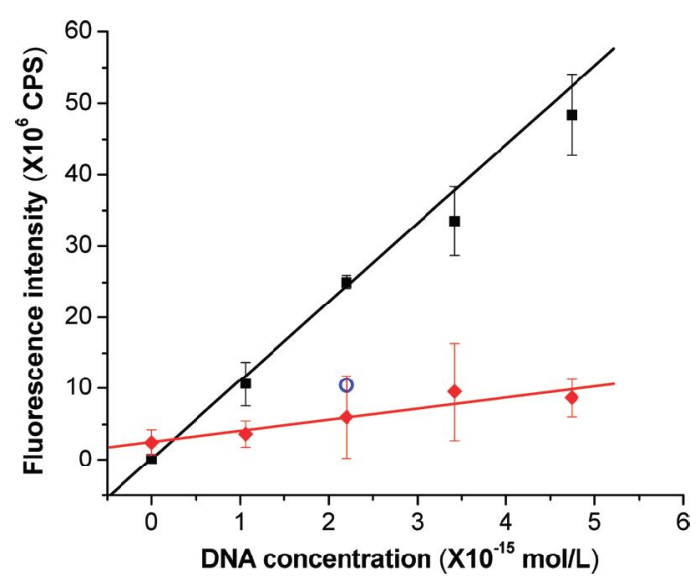

Figure 7. Calibration curves for 20-mer single-stranded DNA targets complexed with target-ready NPs measured by IFC: calibration curve in black (filled squares) for the perfectly matched target analyte and in red (filled diamonds) for the two-mismatch sequence. The open circle symbol indicates the fluorescence signal recorded for a 100 equiv excess of the two-mismatch sequence. The limit of detection for the perfectly matched 20-mer target is $1 \times 10^{4}$ molecules, or $20 \mathrm{zmol}$ in a volume of $20 \mu \mathrm{L}$. calculated as $\sim 1 \times 10^{4}$ molecules, or $2 \times 10^{-20} \mathrm{~mol}$ in the $20 \mu \mathrm{L}$ sample aliquot measured by IFC, or $8 \times 10^{-16}$ M. In comparison with the perfect hybridization, the presence of sequences having two mismatches leads only to a slight increase of luminescence, as does the addition of a large excess (100 equiv) of the twomismatch oligonucleotides. The presence of large excesses of noncomplementary DNA (e.g., fragmented human genomic DNA) can in principle interfere with the polymeric transducer because, though the probe may be designed to be unique, numerous loci presenting one or a few mismatches with the probe are still possible. Remarkably, Ho et al. succeeded in distinguishing a specific 20-mer sequence from fragmented human genomic DNA, despite the presence in the entire genome of numerous one- or two-mismatch loci. ${ }^{36}$ This high selectivity toward the ssDNA target is granted by the very high stringency used during the detection step, that is, in pure water at $60^{\circ} \mathrm{C}$, in which hybridization essentially only occurs with the ssDNA probes electrostatically bound with the cationic 
polythiophene chains within the target-ready NP aggregates, which act as the only counterion available for the negative charges of the phosphate moieties. On the basis of the prior work of Doré et al., the signal generated from the hybridization of a single-mismatch sequence (single nucleotide polymorphism, SNP) should be higher than that of the two-mismatch sequence. ${ }^{34}$ Interestingly, the slower hybridization kinetics of single-mismatch sequences versus perfectly matched targets also reported by Doré et al. could in principle be exploited in a flow cytometry detection scheme to increase selectivity, by optimizing the time separating the addition of targets to the nanoprobes and the measurement of fluorescence. The introduction of mismatched target DNA does not seem to perturb the NP aggregates, given the fact that their size remains relatively unchanged. However, the average luminosity of individual aggregates is much lower, an indication that only perfectly matched oligonucleotides induce adequate energy transfer (Supporting Information, Figure S9). Moreover, the analytical signal measured for complementary targets was found to be linear up to $\sim 10^{-12} \mathrm{M}\left(\sim 6 \times 10^{5}\right.$ copies $/ \mu \mathrm{L}$ ) and to decrease at higher concentrations (Supporting Information, Figure S10). Considering the large number of probes available on each nanoparticle ( $\sim 1500 / \mathrm{NP}$ or $\left.\sim 6 \times 10^{8} / \mu \mathrm{L}\right)$, the particle capacity is not expected to be a limiting factor. Rather, the departure from linearity observed at higher concentration seems to result from the disruption of the NP aggregates due to increasing electrostatic repulsions within them. This was confirmed by IFC (Supporting Information, Figure S10) and dark-field scattering microscopy experiments (Supporting Information, Figure S7) which show a significant decrease in spot size for higher target concentrations. Since it was shown that the majority of the luminescence is emitted from these larger aggregates, their suppression is expected to cause a severe drop in sensitivity.

\section{CONCLUSION}

We have presented herein a DNA sensing architecture combining the molecular recognition capabilities of a cationic conjugated polymer transducer with highly fluorescent core-shell NPs and featuring a unique amplification mechanism which is the result of various essential elements. The very structure of the probe-labeled core-shells NPs and the resulting polymer-induced aggregates maximize the proximity between donors and acceptors that is required for optimal RET. Moreover, strong plasmonic coupling between metal cores is responsible for the enhanced luminosity of fluorescent species as well as the increased range and efficiency of RET, favoring the excitation of several acceptors by each polymer donor throughout the aggregate system. Finally, due to the acceptor's small Stokes shift, homotransfer between eosin molecules can promote energy distribution to the entire structure. Moreover, each hybridization event is signaled by a large number of excited reporters following the efficient plasmon-mediated energy transfer between target-activated polymer transducer and the numerous fluorophores located in the self-assembled core-shell aggregates. The combination of both events results in the sensitive detection of target nucleic acids at femtomolar concentrations. Given these favorable characteristics, such target-ready NP aggregates could allow ultrasensitive and sequence-specific sensing of minute amounts of DNA from aqueous sample volumes, without any labeling or amplification of the nucleic acids.

\section{METHODS}

Materials. The cationic conjugated polymer (CCP), poly $(1 \mathrm{H}$ imidazolium, 1-methyl-3-[2-[(4-methyl-3-thienyl)oxy]ethyl]-, chloride), was graciously provided by the group of Prof. Mario Leclerc and synthesized according to the procedure previously published. ${ }^{33}$ Silver nitrate $\left(\mathrm{AgNO}_{3}\right.$, Aldrich), sodium citrate tribasic dihydrate (Na-Cit, Aldrich), tetraethoxysilane (TEOS, Aldrich), ammonia ( $\mathrm{NH}_{4} \mathrm{OH} 30 \%$, Aldrich), eosin isothiocyanate (EiTC, Marker Gene Technologies), 3-(aminopropyl)triethoxysilane (APS, Aldrich), succinic anhydride (SA, Aldrich), N-hydroxysuccinimide (NHS, Aldrich), and sodium chloride $(\mathrm{NaCl}$, Aldrich) were used as received. Deionized water (18 $\mathrm{M} \Omega$ ), anhydrous ethanol (EtOH), N,N-dimethylformamide anhydrous (DMF, Aldrich), and 2-( $\mathrm{N}$-morpholino)ethanesulfonic acid buffer (MES, Aldrich) were used as dispersion media.

DNA Sequences. Oligonucleotides were all purchased from Integrated DNA Technologies, Inc. (Coralville, IA) in a standard desalting purification grade, except for the Cy5.5-labeled probe, which was HPLC purification grade. The oligonucleotide sequences used in this work are shown in Table S1 (Supporting Information). An amino-modified 20-mer sequence corresponding to a conserved region of the Candida albicans yeast genome ${ }^{34}$ was used as the capture probe. The binding capacity of the probe-functionalized fluorescent core-shell NPs was determined using the same probe sequence labeled at the $3^{\prime}$ end with a Cy5.5 dye.

\left. Synthesis of Silver Nanoparticles, Spacer Silica Shell ( ${\mathrm{Ag} @ S i O_{2}}_{2}\right)$, EiTCDoped Silica Shell (Ag@SiO ${ }_{2} @ S_{2} O_{2}+$ EiTC), and CCP/Ag@SiO ${ }_{2}$ Self-Assemblies. The method employed for preparation of fluorescent multilayer core-shell NPs was described elsewhere. ${ }^{22}$ Typically, a silver colloid with an average particle size of $50 \pm 10 \mathrm{~nm}$ was prepared using a citrate reduction, and a first silica spacer shell (7 $\pm 1 \mathrm{~nm}$ ) was grown on the silver nanoparticles using an adaptation of the Stöber method. The particle concentration of the colloidal solution, at $\sim 2 \times 10^{10} / \mathrm{mL}$, was obtained from the determination of elemental Ag by flame atomic absorption spectrometry and calculated for an average diameter of $50 \mathrm{~nm}$. The eosin acceptor dye was covalently incorporated in a second silica shell using a dye-conjugated silane coupling agent. The thickness of the silica spacer and the dye concentration were optimized to find the optimal fluorescence signal in terms of metal-fluorophore distance and self-quenching between eosin molecules. These fluorescent nanoparticles were used either as-is for spectroscopic measurements or for 
the preparation of probe-grafted fluorescent core-shell nanocomposites.

For evaluation of the CCP fluorescence enhancement factor, $\mathrm{CCP} / \mathrm{Ag} @ \mathrm{SiO}_{2} \mathrm{NPs}$ were self-assembled by exploiting the electrostatic attractions between positively charged CCPs and the negatively charged surfaces of the core-shell NPs. Typically, $20 \mu \mathrm{L}$ of $8.4 \times 10^{-4} \mathrm{M} \mathrm{CCP}$ solution (calculated on a monomer unit basis) was added to a $1 \mathrm{~mL}$ NP aliquot and shaken manually for $30 \mathrm{~min}$ at room temperature. The resulting CCP/Ag@SiO NPs were centrifuged and washed three times with water to remove the excess of CCP and then dispersed in $1 \mathrm{~mL}$ of water. The procedure used to prepare hollow silica nanoshell control samples from the fluorescent core-shell nanoparticles was described elsewhere (see Figures S2 and S3 in Supporting Information). ${ }^{22}$

Surface Modification and DNA Functionalization. The bioconjugation of the as-prepared fluorescent core-shell NPs with ssDNA probes was performed by amine coupling processes. First, amine-functionalized NPs were prepared by covalent surface grafting of APS. To do so, $50 \mu \mathrm{L}$ of APS was added to a $10 \mathrm{~mL}$ aliquot of fluorescent NPs $\left(2 \times 10^{10} \mathrm{NPs} / \mathrm{mL}\right)$ and stirred for $2 \mathrm{~h}$. After that, the NPs were collected by centrifugation $(10000 \mathrm{rpm}$ $25 \mathrm{~min}$ ) and washed three times with $\mathrm{EtOH}$, and then redispersed in $5 \mathrm{~mL}$ of anhydrous DMF. Succinic anhydride was used to convert the amine functional groups into carboxylate functions. To $5 \mathrm{~mL}$ of fluorescent core-shell nanoparticles was added $1 \mathrm{~mL}$ of a $50 \mathrm{mM}$ succinic anhydride solution in DMF, and the mixture was stirred for $4 \mathrm{~h}$ at room temperature. The carboxylate-functionalized NPs were collected by centrifugation and washed three times with water and redispersed in $5 \mathrm{~mL}$ of MES buffer solution ( $10 \mathrm{mM}, \mathrm{pH}$ 6). NHS-ester activation chemistry was then used to bind the amino-terminated oligonucleotides onto the carboxylate-modified NPs. Briefly, an NHSester derivative is formed by reacting carboxylate groups with NHS. Stable amide linkages are obtained by nucleophilic reaction between the amine compounds and the carbonyl of the active esters. In our case, the conjugation step was performed by mixing $1 \mathrm{nmol}$ of amino-terminated capture probe to $5 \mathrm{~mL}$ of carboxylate-modified NPs, followed by the addition of $50 \mu \mathrm{L}$ of a $50 \mathrm{mM}$ NHS solution. The mixture was let to react $12 \mathrm{~h}$ in the dark at $4{ }^{\circ}$ C. EDC (1-ethyl-3-[3-dimethylaminopropyl]carbodiimide hydrochloride), which is commonly used as a crosslinking agent in NHS chemistry, was not used in this conjugation step to avoid partial erosion of the silver metallic core by chloride ions. Instead, a longer reaction time was used and a slight increase in $\mathrm{pH}(6.0-6.5)$ promoted nucleophilic attack by the amine groups in their more active nonprotonated form. The oligo-functionalized fluorescent NPs were collected by centrifugation and washed three times with water and were finally redispersed in $5 \mathrm{~mL}$ of EtOH to avoid sample degradation. The resulting oligonucleotide-nanoparticle conjugates were stored at $4{ }^{\circ} \mathrm{C}$ and remained stable for months.

The binding capacity of the probe-functionalized fluorescent core-shell NPs was determined experimentally by measuring the amount of Cy5.5-labeled probe left in the supernatant after the conjugation step with the NPs. The results show that $\sim 1500$ hybridization sites can be found on average per probe-functionalized NP.

Sample Preparation and Hybridization Conditions. In order to prepare target-ready NPs, $250 \mu \mathrm{L}$ aliquots of probe-grafted NPs in EtOH were centrifuged and resuspended in $250 \mu \mathrm{L}$ of water. To prevent interference on the polythiophene transducer by other cationic counterions, all reactions and experiments were carried out in electrolyte-free $18 \mathrm{M} \Omega$ nanopure water. Then, $15 \mu \mathrm{L}$ of a $8.4 \times 10^{-6} \mathrm{M}$ CCP solution (calculated on a monomer unit basis) was added to the NPs and shaken manually for $30 \mathrm{~min}$ at room temperature. These CCP/probe NPs (i.e., target-ready NPs) were then centrifuged and washed three times with water to remove any unbound CCP and redispersed in $250 \mu \mathrm{L}$ of water. For the target detection experiments, a $3 \times 10^{8} \mathrm{NPs} / \mathrm{mL}$ suspension was prepared by diluting $30 \mu \mathrm{L}$ of target-ready NPs in $1.5 \mathrm{~mL}$ of water. The complementary target oligonucleotides were added to the target-ready NP suspension, the hybridization was allowed to proceed at $60^{\circ} \mathrm{C}$ for $10 \mathrm{~min}$, and $20 \mu \mathrm{L}$ aliquots of the hybridized NPs were presented to the imaging flow cytometry apparatus. In this system, the electrostatically bound CCP acts as a localized counterion to the negative phosphate moieties to promote hybridization in pure water, that is, without salt. ${ }^{36}$ The calibration curves were obtained by measuring the fluorescence signal for successive additions of target oligonueotides, after the specified $10 \mathrm{~min}$ equilibration period. Since aggregate formation occurs over a period of time, control over reaction and equilibration times is necessary to obtain reproducible detection sensitivity. The detection limit was calculated as 3 times the standard deviation of the fluorescence signal for the blank (in the present case, the solution with the lowest target concentration used to establish the calibration curve) divided by the slope of the curve. Note that the as-prepared probe-grafted fluorescent Ag@SiO 2 nanoprobes are very stable at elevated temperatures and neither a shift or broadening of the absorption peak nor a reduction of fluorescence was observed after a long heating period.

It should be noted that target-ready NPs must be prepared using an excess of DNA probes compared to CCP. Because of the strong positively charged nature of the CCP (one positive charge per monomer unit) and the anionic character of the probe-functionalized NPs, significant nonspecific adsorption is observed when an excess of CCP is added to the probe-grafted NPs. The normally nonfluorescent target-ready NPs then become highly fluorescent since a non-negligible fraction of the CCP exists in a non-DNA-complexed, fluorescent random coil conformation.

Acknowledgment. This work was supported by the Natural Sciences and Engineering Research Council of Canada, the "Fonds Québécois de la Recherche sur la Nature et les Technologies du Québec", the Canadian Foundation for Innovation, Héma-Québec, and the Canadian Blood Services. The authors would like to acknowledge the contributions of Marie-Pier Blais for the cover artwork, Maryse St-Louis (Héma-Québec) and Dr. Kim Doré (CRULRG, Université Laval) for stimulating discussions, Prof. Katherine Willets (Department of Chemistry, University Texas at Austin) for useful advice on dark-field microscopy, and Prof. Mario Leclerc and his group (Department of Chemistry, Universite Laval) for the gift of the polymer and a long-standing collaboration on polymer biosensors.

Supporting Information Available: IFC method and other data, fluorescence spectra of core-shell nanoparticles and polymer transducer, TEM and dark-field images. This material is available free of charge via the Internet at http://pubs.acs.org.

\section{REFERENCES AND NOTES}

1. Brettell, T. A.; Butler, J. M.; Almirall, J. R. Forensic Science. Anal. Chem. 2009, 81, 4695-4711.

2. Palacios, G.; et al.Panmicrobial Oligonucleotide Array for Diagnosis of Infectious Diseases. Emerg. Infect. Dis. 2007, 13, 73-81.

3. Deligeorgiev, T. G.; Kaloyanova, S.; Vaquero, J. J. Intercalating Cyanine Dyes for Nucleic Acid Detection. Recent Pat. Mater. Sci. 2009, 2, 1-26.

4. Dylla-Spears, R.; Townsend, J. E.; Sohn, L. L.; Jen-Jacobson, L.; Muller, S. J. Fluorescent Marker for Direct Detection of Specific dsDNA Sequences. Anal. Chem. 2009, 81, 1004910054.

5. Meng, X.; Yang, X.; Wang, K.; Guo, Q.; Tan, Y.; Mo, Q.; Xu, X. Direct Fluorescence Detection of Point Mutations in Human Genomic DNA Using Microbead-Based Ligase Chain Reaction. Talanta 2010, 80, 1725-1729.

6. Summersgill, B.; Clark, J.; Shipley, J. Fluorescence and Chromogenic In Situ Hybridization To Detect Genetic Aberrations in Formalin-Fixed Paraffin Embedded Material, Including Tissue Microarrays. Nat. Protoc. 2008, 3, 220-234.

7. Vora, G. J.; Meador, C. E.; Anderson, G. P.; Taitt, C. R. Comparison of Detection and Signal Amplification Methods for DNA Microarrays. Mol. Cell. Probes 2008, 22, 294-300.

8. Dalavoy, T. S.; Wernette, D. P.; Gong, M.; Sweedler, J. V.; Lu, Y.; Flachsbart, B. R.; Shannon, M. A.; Bohn, P. W.; Cropek, D. M. Immobilization of DNAzyme Catalytic Beacons on PMMA for $\mathrm{Pb}^{2+}$ Detection. Lab Chip 2008, 8, 786-793. 
9. Bustin, S. A. Quantification of mRNA Using Real-Time Reverse Transcription PCR (RT-PCR): Trends and Problems. J. Mol. Endocrinol. 2002, 29, 23-39.

10. Bustin, S. A.; Benes, V.; Nolan, T.; Pfaffl, M. W. Quantitative Real-Time RT-PCR: A Perspective. J. Mol. Endocrinol. 2005, 34, 597-601.

11. Murphy, J.; Bustin, S. Real-Time Quantitative PCR as a Bioanalytical Tool in the Pharmaceutical Industry. Int. Drug Discovery 2010, 5, 12-20.

12. Halford, W. P. The Essential Prerequisites for Quantitative RT-PCR. Nat. Biotechnol. 1999, 17, 835.

13. Wilke, W. W.; Jones, R. N.; Sutton, L. D. Automation of Polymerase Chain Reaction Tests: Reduction of Human Errors Leading to Contamination. Diagn. Microbiol. Infect. Dis. 1995, 21, 181-185.

14. Saiki, R. K.; Scharf, S.; Faloona, F.; Mullis, K. B.; Horn, G. T.; Erlich, H. A.; Arnheim, N. Enzymatic Amplification of Beta-Globin Genomic Sequences and Restriction Site Analysis for Diagnosis of Sickle Cell Anemia. Science 1985, 230, 1350-1354.

15. Fan, X.; White, I. M.; Shopova, S. I.; Zhu, H.; Suter, J. D.; Sun, Y. Sensitive Optical Biosensors for Unlabeled Targets: A Review. Anal. Chim. Acta 2008, 620, 8-26.

16. Doré, K.; Leclerc, M.; Boudreau, D. Fluorescence Signal Amplification for Ultrasensitive DNA Detection. Rev. Fluoresc. 2009, Volume 2007, 179-197.

17. Yao, G.; Wang, L.; Wu, Y.; Smith, J.; Xu, J.; Zhao, W.; Lee, E.; Tan, W. FloDots: Luminescent Nanoparticles. Anal. Bioanal. Chem. 2006, 385, 518-524.

18. Imhof, A.; Megens, M.; Engelberts, J. J.; De Lang, D. T. N Sprik, R.; Vos, W. L. Spectroscopy of Fluorescein (FITC) Dyed Colloidal Silica Spheres. J. Phys. Chem. B 1999, 103, 1408-1415.

19. Gill, R.; Zayats, M.; Willner, I. Semiconductor Quantum Dots for Bioanalysis. Angew. Chem., Int. Ed. 2008, 47, 7602 7625.

20. Smith, J. E.; Medley, C. D.; Tang, Z.; Shangguan, D.; Lofton, C.; Tan, W. Aptamer-Conjugated Nanoparticles for the Collection and Detection of Multiple Cancer Cells. Anal. Chem. 2007, 79, 3075-3082.

21. Viger, M. L.; Live, L. S.; Therrien, O. D.; Boudreau, D. Reduction of Self-Quenching in Fluorescent Silica-Coated Silver Nanoparticles. Plasmonics 2008, 3, 33-40.

22. Lessard-Viger, M.; Rioux, M.; Rainville, L.; Boudreau, D. FRET Enhancement in Multilayer Core-Shell Nanoparticles. Nano Lett. 2009, 9, 3066-3071.

23. Stranik, O.; Nooney, R.; McDonagh, C.; MacCraith, B. D. Plasmonic Enhancement Using Core-Shell Nanoparticles. Proc. SPIE: Int. Soc. Opt. Eng. 2005, 5824, 79-85.

24. Aslan, K.; Wu, M.; Lakowicz, J. R.; Geddes, C. D. Fluorescent Core-Shell Ag@SiO, Nanocomposites for MetalEnhanced Fluorescence and Single Nanoparticle Sensing Platforms. J. Am. Chem. Soc. 2007, 129, 1524-1525.

25. Tovmachenko, O. G.; Graf, C.; van den Heuvel, D. J.; van Blaaderen, A.; Gerritsen, H. C. Fluorescence Enhancement by Metal-Core/Silica-Shell Nanoparticles. Adv. Mater. 2006, 18, 91-95.

26. Cheng, D.: Xu, Q.-H. Separation Distance Dependent Fluorescence Enhancement of Fluorescein Isothiocyanate by Silver Nanoparticles. Chem. Commun. 2007, 248-250.

27. Bardhan, R.; Grady, N. K.; Cole, J. R.; Joshi, A.; Halas, N. J. Fluorescence Enhancement by Au Nanostructures: Nanoshells and Nanorods. ACS Nano 2009, 3, 744-752.

28. Martini, M.; Perriat, P.; Montagna, M.; Pansu, R.; Julien, C.; Tillement, O.; Roux, S. How Gold Particles Suppress Concentration Quenching of Fluorophores Encapsulated in Silica Beads. J. Phys. Chem. C 2009, 113, 17669 17677.

29. Reil, F.; Hohenester, U.; Krenn, J. R.; Leitner, A. Forster-Type Resonant Energy Transfer Influenced by Metal Nanoparticles. Nano Lett. 2008, 8, 4128-4133.

30. Zhang, J.; Fu, Y.; Chowdhury, M. H.; Lakowicz, J. R. Enhanced Foerster Resonance Energy Transfer on Single Metal Particle. 2. Dependence on Donor-Acceptor Separation Distance, Particle Size, and Distance from Metal Surface. J. Phys. Chem. C 2007, 111, 11784-11792.
31. Zhang, J.; Fu, Y.; Lakowicz, J. R. Enhanced Foerster Resonance Energy Transfer (FRET) on a Single Metal Particle. J. Phys. Chem. C 2007, 111, 50-56.

32. Xie, H. Y.; Chung, H. Y.; Leung, P. T.; Tsai, D. P. Plasmonic Enhancement of Forster Energy Transfer between Two Molecules in the Vicinity of a Metallic Nanoparticle: Nonlocal Optical Effects. Phys. Rev. B 2009, 80, 155448/ $1-155448 / 10$

33. Ho, H.-A.; Boissinot, M.; Bergeron, M. G.; Corbeil, G.; Doré, K.; Boudreau, D.; Leclerc, M. Colorimetric and Fluorometric Detection of Nucleic Acids Using Cationic Polythiophene Derivatives. Angew. Chem., Int. Ed. 2002, 41, 1548-1551.

34. Doré, K.; et al.Fluorescent Polymeric Transducer for the Rapid, Simple, and Specific Detection of Nucleic Acids at the Zeptomole Level. J. Am. Chem. Soc. 2004, 126, 4240 4244.

35. Liu, B.; Bazan, G. C. Homogeneous Fluorescence-Based DNA Detection with Water-Soluble Conjugated Polymers. Chem. Mater. 2004, 16, 4467-4476.

36. Ho, H. A.; Doré, K.; Boissinot, M.; Bergeron, M. G.; Tanguay, R. M.; Boudreau, D.; Leclerc, M. Direct Molecular Detection of Nucleic Acids by Fluorescence Signal Amplification. J. Am. Chem. Soc. 2005, 127, 12673-12676.

37. Gaylord, B. S.; Heeger, A. J.; Bazan, G. C. DNA Detection Using Water-Soluble Conjugated Polymers and Peptide Nucleic Acid Probes. Proc. Natl. Acad. Sci. U.S.A. 2002, 99, 10954-10957.

38. Wang, Y.; Liu, B. Silica Nanoparticle Assisted DNA Assays for Optical Signal Amplification of Conjugated Polymer Based Fluorescent Sensors. Chem. Commun. 2007, 3553 3555.

39. Doré, K.; Leclerc, M.; Boudreau, D. Investigation of a Fluorescence Signal Amplification Mechanism Used for the Direct Molecular Detection of Nucleic Acids. J. Fluoresc. 2006, 16, 259-265.

40. Xia, F.; et al.Colorimetric Detection of DNA, Small Molecules, Proteins, and lons Using Unmodified Gold Nanoparticles and Conjugated Polyelectrolytes. Proc. Natl. Acad. Sci. U.S.A. 2010, 107, S10837/1-S10837/5.

41. Elghanian, R.; Storhoff, J. J.; Mucic, R. C.; Letsinger, R. L.; Mirkin, C. A. Selective Colorimetric Detection of Polynucleotides Based on the Distance-Dependent Optical Properties of Gold Nanoparticles. Science 1997, 277, 1078 1080.

42. Zhang, J.; Fu, Y.; Chowdhury, M. H.; Lakowicz, J. R. MetalEnhanced Single-Molecule Fluorescence on Silver Particle Monomer and Dimer: Coupling Effect between Metal Particles. Nano Lett. 2007, 7, 2101-2107.

43. Zhang, J.; Fu, Y.; Chowdhury, M. H.; Lakowicz, J. R. SingleMolecule Studies on Fluorescently Labeled Silver Particles: Effects of Particle Size. J. Phys. Chem. C 2008, 112, 18-26.

44. Liu, S.; Han, M. Synthesis, Functionalization, and Bioconjugation of Monodisperse, Silica-Coated Gold Nanoparticles: Robust Bioprobes. Adv. Funct. Mater. 2005, 15, 961967.

45. Liu, S.; Zhang, Z.; Han, M. Gram-Scale Synthesis and Biofunctionalization of Silica-Coated Silver Nanoparticles for Fast Colorimetric DNA Detection. Anal. Chem. 2005, 77, 2595-2600.

46. Dubus, S.; Gravel, J.-F.; Le Drogoff, B.; Nobert, P.; Veres, T.; Boudreau, D. PCR-Free DNA Detection Using a Magnetic Bead-Supported Polymeric Transducer and Microelectromagnetic Traps. Anal. Chem. 2006, 78, 4457-4464.

47. Geddes, C. D., Lakowicz, J. R., Eds. Radiative Decay Engineering. In Topics in Fluorescence Spectroscopy; Springer: New York, 2005; Vol. 8, 457 pp.

48. Lakowicz, J. R. Principles of Fluorescence Spectroscopy, 2nd ed.; Plenum Publishing Corporation: New York, 1999.

49. Tang, F.; He, F.; Cheng, H.; Li, L. Self-Assembly of Conjugated Polymer-Ag@SiO 2 Hybrid Fluorescent Nanoparticles for Application to Cellular Imaging. Langmuir 2010, 26, 11774-11778.

50. Pan, S.; Rothberg, L. J.; Nolte, A. J.; Rubner, M. F.; Gorodetskaya, I.; Swager, T. M. Plasmon-Enhanced Conjugated Polymer Luminescence Using Silver Nanoparticles and 
Sequentially Adsorbed Polyelectrolyte Spacers. Proc. SPIE: Int. Soc. Opt. Eng. 2005, 5927, 592705/1-592705/8.

51. Park, H.-J.; Vak, D.; Noh, Y.-Y.; Lim, B.; Kim, D.-Y. Surface Plasmon Enhanced Photoluminescence of Conjugated Polymers. Appl. Phys. Lett. 2007, 90, 161107/1-161107/3.

52. Li, H.; Rothberg, L. Colorimetric Detection of DNA Sequences Based on Electrostatic Interactions with Unmodified Gold Nanoparticles. Proc. Natl. Acad. Sci. U.S.A. 2004, 101, 14036-14039.

53. Liu, Z.; Wang, H.; Li, H.; Wang, X. Red Shift of Plasmon Resonance Frequency Due to the Interacting Ag Nanoparticles Embedded in Single Crystal $\mathrm{SiO}_{2}$ by Implantation. Appl. Phys. Lett. 1998, 72, 1823-1825.

54. Hu, M.; Ghoshal, A.; Marquez, M.; Kik, P. G. Single Particle Spectroscopy Study of Metal-Film-Induced Tuning of Silver Nanoparticle Plasmon Resonances. J. Phys. Chem. C 2010, 114, 7509-7514.

55. Ringler, M.; Schwemer, A.; Wunderlich, M.; Nichtl, A.; Kuerzinger, K.; Klar, T. A.; Feldmann, J. Shaping Emission Spectra of Fluorescent Molecules with Single Plasmonic Nanoresonators. Phys. Rev. Lett. 2008, 100, 203002/ $1-203002 / 4$. 